RECAI

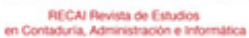

RECAI Revista de Estudios en Contaduría, Administración e Infomática

ISSN: 2007-5278

mrdemunerf@uaemex.mx

Universidad Autónoma del Estado de México

México

\title{
Análisis de la calidad del servicio en IES a través del modelo SERVQUAL
}

Barragán Vázquez, Carlos Hugo; García Martínez, J. Jesús; Medina Heredia, Nancy Vanessa Análisis de la calidad del servicio en IES a través del modelo SERVQUAL

RECAI Revista de Estudios en Contaduría, Administración e Infomática, vol. 11, núm. 30, 2022

Universidad Autónoma del Estado de México, México

Disponible en: https://www.redalyc.org/articulo.oa? $\mathrm{id}=637969396001$ 
Artículos

\title{
Análisis de la calidad del servicio en IES a través del modelo SERVQUAL
}

\author{
Analysis of the service quality in IES through the \\ SERVQUAL model \\ Carlos Hugo Barragán Vázquez carloshb@ucol.mx \\ Universidad de Colima, México \\ J. Jesús García Martínez jgarciam@ucol.mx \\ Universidad de Colima, México \\ Nancy Vanessa Medina Heredia medina_heredia@ucol.mx \\ Universidad de Colima, México
}

RECAI Revista de Estudios en

Contaduría, Administración e Infomática, vol. 11, núm. 30, 2022

Universidad Autónoma del Estado de México, México

Recepción: 01 Agosto 2021 Aprobación: 26 Noviembre 2021

Redalyc: https://www.redalyc.org/ articulo.oa?id $=637969396001$
Resumen: El presente artículo tiene como objetivo evaluar la calidad del servicio de un proceso administrativo realizado en las Instituciones de Educación Superior: el Servicio Social Constitucional. La investigación es de tipo cuantitativa, con un enfoque descriptivo correlacional, en la cual, se utilizó el modelo SERVQUAL para medir la calidad percibida por la comunidad estudiantil. Se aplicó la encuesta a 425 estudiantes del campus central de la Universidad de Colima. Los datos obtenidos fueron analizados por distintas técnicas, correlación de Pearson, Aalfa de Cronbach y regresión lineal multinominal. Los resultados muestran que, para los usuarios, la calidad del servicio es significativa en las dimensiones de elementos tangibles y empatía de los servicios.

Palabras clave: calidad del servicio, Servqual, educación superior y regresión lineal.

Abstract: The objective of this article was to evaluate the service quality of an administrative process carried out in higher education institutions: The Constitutional Social Service. This research approach was quantitative, with a descriptive-correlational focus, in which the SERVQUAL model was used to measure the quality perceived by the student community. A survey was chosen as the data collection instrument and it was administered to 425 students at the central campus of the University of Colima. The data obtained were analyzed using different techniques, Pearson's correlation, Cronbach's alpha, and multinomial linear regression. The results show that, for users, the quality of the service was significant in the dimensions of tangible elements and service empathy. Keywords: service quality, Servqual, higher education and line regression.

\section{INTRODUCCIÓN}

Evaluar la calidad del servicio de instituciones universitarias es una constante preocupación en el actual sistema educativo y sobre todo una tarea que atañe a aquellos agentes que tienen la responsabilidad de planear y proyectar el destino de las instituciones del superior sobre todo en el ámbito público. En México esta temática se ha tornado un aspecto fundamental para garantizar la calidad educativa, de ahí de ahí que se haga imperante trae evaluar la satisfacción de los clientes en las entidades educativas. Una de estas metodologías de evaluación es mediante el modelo SERQUAL, como que se aplica para evaluar los servicios conforme a las expectativas de los usuarios, así como garantizar que los procedimientos cumplan con las metas señaladas en la planeación. 
De acuerdo con Geraldo (2020), para llevar a cabo un proceso de calidad es de suma importancia que se cuente con un soporte de liderazgo sólido que promueva en la organización el involucramiento y la participación total en la toma de decisiones de todas las jerarquías existentes. El concepto de calidad ha pasado por varias etapas a lo largo de la historia que, desde la perspectiva educacional, la implementación de modelos y métodos desarrollados a la fecha para medir y evaluar la calidad han logrado un impacto trascendental en las universidades, lo que abona al desarrollo integral de la formación del estudiante.

La ausencia de una evaluación, en el caso particular del proceso de acreditación del Servicio Social Constitucional (SSC), genera un estancamiento de eficacia, fluidez y mejoramiento al servicio de calidad que se pretende brindar al estudiante. En base a esto, Sotelo y Figueroa (2017) reafirman la importancia que ello implica, toda vez que, a través de la evaluación se podrá obtener el nivel de calidad con el cual se desarrollan y trabajan las actividades que se llevan a cabo mediante un control específico, determinando si el proceso es adecuado o incompleto. Por lo tanto, el tener un control en un proceso administrativo actual, no significa que esté funcionando a la perfección o que no pueda sufrir modificaciones para implementar una mejora en el mismo y que impacte en la calidad institucional.

En este sentido, el objetivo de esta investigación es evaluar los factores de la calidad en el servicio del proceso de acreditación del Servicio Social Constitucional, conforme al modelo SERVQUAL (tangibles, la respuesta, la entrega, la empatía y la seguridad). Los resultados permitirán implementar una mejora continua en procesos administrativos de una institución de nivel superior en México, enfocada a conocer el nivel de calidad del servicio en base a la percepción del estudiante como mayor consumidor. La evaluación está dirigida al proceso de acreditación de una de sus materias formativas: el SSC, a través de uno de los modelos de calidad con más realce en el ámbito educacional, y que, con los resultados obtenidos, se abone a la identificación acertada de áreas de oportunidad en beneficio del personal académico, administrativo y estudiantil; con la mejora en eficacia y eficiencia de la optimización de un proceso impactando en la calidad institucional.

\section{REFERENTE CONCEPTUAL}

\section{Calidad de la educación superior}

Recientemente el interés por los temas de la calidad educativa ha incrementado el índice de investigaciones, como consecuencia de las demandas económicas, políticas y sociales que requieren el involucramiento activo de las instituciones de educación superior en el desarrollo económico de los países (Pérez-Cruz, 2016). En ese sentido Reyes, Nande y Hernández (2020) sostienen que es necesario identificar los factores que influyen en el involucramiento de las universidades en el contexto regional y nacional. Así, la evaluación de la calidad educativa 
constituye una de las mejores estrategias para el fortalecimiento de las universidades pues su análisis se relaciona directa directamente con los procesos curriculares, la acreditación académica y la vinculación de las universidades (Perez-Cruz \& Pinto-Perez, 2020).

Un consenso sobre la calidad es que es entendida de manera general como la satisfacción de las necesidades y/o expectativas de los clientes/ usuarios. Al respecto Cardona y Bravo (2012) definen la calidad como conjunto de características inherentes a un producto o servicio que le confieren la capacidad de atender los requerimientos explícitos e implícitos de los usuarios o clientes. En tal sentido Flores y Perez (2006) afirman que el prestar un servicio se tiene que identificar las necesidades de los usuarios en este caso sobre la oferta educativa esta misma afirmación se transporta hacia el ámbito educativo para el cumplimiento de los procesos y necesidades implícitas del sistema educativo. Es así que la calidad se entiende como la satisfacción de todas las partes interesadas, integrando tanto al personal administrativo y de apoyo, como al que hasta en contacto con los clientes, los intermediarios hasta llegar a los usuarios finales.

La perspectiva sobre la calidad educativa y en especial sobre la calidad en instituciones de educación superior es muy amplia. No obstante, el concepto de educación de calidad se ha consolidado a partir de la teoría del capital humano, haciendo hincapié en la formación del del personal como parte fundamental del eslabón económico para el crecimiento y bienestar de los países. Autores como Licona-Michel y Perez-Cruz (2018) sostienen que el capital humano es parte es una parte fundamental del análisis económico de los países, y que ha demostrado ser un elemento importante del crecimiento y desarrollo económico al identificar la educación como elemento que provee al futuro trabajador de las habilidades y herramientas necesarias para desempeñarse de esta manera insertarse en el mercado laboral y obtener mejores remuneraciones y por consiguiente aumentar su calidad de vida.

La relación entre la calidad educativa, el bienestar social y el desarrollo económico ha sido ampliamente demostrada por las investigaciones que evidencian este vínculo entre el crecimiento económico y el acceso a la educación superior como vía de acceso para las trayectorias laborales (Perez-Cruz, 2018). En este sentido la calidad educativa ya no se centra en aspectos tangibles la población estudiantil, el claustro docente o el número de egresos entre otros aspectos; sino que se busca analizar el aprendizaje, el escenario y su contexto inmediato y de largo alcance. Al respecto, Pérez-Cruz (2019) y Cervantes (2017) explican que el principal reto de la educación superior a nivel mundial, es la búsqueda de la calidad, relacionada de manera directa con el estudiante, es decir una educación centrada en el estudiante, donde éste reciba una educación integral mediante tecnologías que sean innovadoras pero a la vez didácticas, buscando la eficiencia de los recursos y la transmisión de conocimientos y desarrollo de habilidades profesionales y personales. 
Parasuraman, Zeithalm, \& Berry (1985) entienden la calidad como una percepción, un juicio de valor que de forma general hace el cliente sobre el servicio o producto que recibe, otorgándole un valor superioridad a las expectativas previas; es decir; la calidad del servicio resulta de comparar el servicio o producto esperado contra el servicio o producto recibido. Montaudon (2010) señala que el modelo SERVQUAL es un poderoso modelo de investigación en administración, que permite obtener de manera profunda, una medición de la calidad en todos sentidos, ya sea ésta para entidades del ámbito educativo, comercial, industrial, manufactura, agropecuario entre otros; lo que permite identificar las expectativas de los clientes y la percepción del servicio obteniendo, generando así información diagnóstica sobre el proceso o servicio que se quiera estudiar.

En ese sentido el modelo teórico SERVQUAL, fue desarrollado por Parasuraman, Zeithalm, \& Berry (1985) y posteriormente fue revisado por sus autores y puesto en práctica en un estudio empírico donde se diseñó la encuesta. Para lo cual, utilizando información de este estudio fueron determinadas las medias y de los estándares de los ítems, así como los coeficientes de confiabilidad para validar los constructos establecidos en dicho modelo, es decir: tangibilidad, fiabilidad, capacidad de respuesta, seguridad y empatía (Parasuraman, Zeithalm, \& Berry, 1988). La siguiente figura muestra estas interacciones.

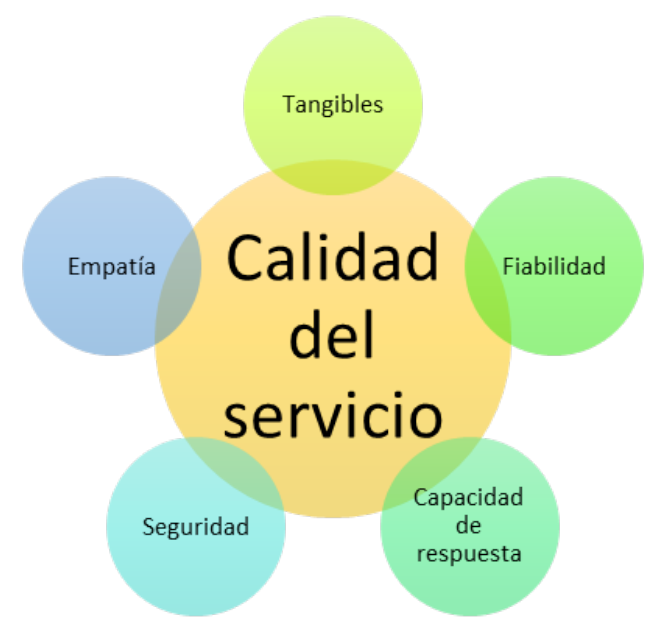

Figura 1

Dimensiones del SERVQUAL

Fuente: Elaboración propia con base en Parasuraman, Zeithalm, \& Berry (1985).

En ese sentido de acuerdo al modelo anterior las 5 dimensiones pueden ser entendidas de diversas maneras acorde a las necesidades del servicio del producto que se pretende evaluar.

1. Los tangibles: son aquellos elementos que integran la parte visible del servicio de producto es decir factores que pueden ser tocados como integrantes del proceso de servicio. 
2. La fiabilidad: es la capacidad que tiene la organización para otorgar un servicio o producto de manera confiable seguro y eficiente; integrando los elementos inherentes al personal que garanticen su correcta entrega.

3. La capacidad de respuesta: hace referencia a la disposición que tiene la empresa para ayudar a sus clientes y resolver problemas de manera oportuna. También integral los compromisos en tiempos de entrega, así como la accesibilidad del cliente para contactar a la empresa.

4. La seguridad: es la percepción que tiene el cliente sobre la atención a sus necesidades y problemáticas, que le confieren la credibilidad en la empresa, para resolver sus necesidades de manera integral confiable y honesta.

5. La empatía: es la disposición de la empresa para brindar una atención personalizada más allá de la cortesía, al conocer a fondo las características y requerimientos específicos del servicio que espera el cliente.

En este sentido, Wang (2015) argumenta que el modelo SERVQUAL es el idóneo para llevar a cabo investigaciones referentes a la medición del nivel de calidad en las IES; aunque existen otros modelos que pueden ser aplicados en el sector de la educación, ya que pueden ser modificados y adaptados a las necesidades requeridas. Así, los modelos que tienen como objetivo evaluar la calidad en un servicio han tenido diversas adaptaciones, esto de acuerdo con la percepción del autor, donde finalmente cada uno tiene en claro la meta: medir la satisfacción del cliente. En el ámbito educativo varias investigaciones apoyan la aplicación de estos modelos, específicamente del SERVQUAL (Duque \& Gómez, 2015).

\section{METODOLOGÍA}

En esta investigación se evaluó la calidad del servicio del proceso de acreditación del servicio social constitucional de la Universidad de Colima. El diseño del método de la presente investigación es correlacional, ya que se miden la relación entre variables, en este caso, la calidad como variable dependiente, y como variables independientes los elementos tangibles, fiabilidad, tiempo de respuesta, seguridad y empatía.

Los individuos que formaron parte del muestro aleatorio, fueron estudiantes del Nivel Superior de la Universidad de Colima (UCOL), que se encuentran actualmente cursando diferentes grados de su plan de estudios, desde el $5^{\circ}$ al $10^{\circ}$ grado, del semestre febrero-agosto 2020 y que corresponden a la Delegación 3 de dicha institución, en la cual se encuentran diversas áreas disciplinares como ciencias de la salud, administración y negocios; artes y humanidades; educación, tecnologías de la información y comunicación; ciencias sociales y derecho; así como ciencias naturales, matemáticas y estadística. El número total de alumnos del Nivel Superior de la Delegación 3 de la UCOL es de 5256, en el semestre en curso febrero-agosto 2020, por lo que, el tamaño de muestra fue de 425 aplicaciones, lo que representó un 96\% de confianza.

El instrumento de recolección de datos seleccionado es una encuesta de escala tipo Likert, basada en el modelo SERVQUAL; la cual establece los indicadores específicos que la investigación requiere, diseñada y adaptada 
de manera personalizada, validada a través de una prueba piloto para proceder con su aplicación. El instrumento estuvo formado por 2020 ítems distribuidas en las 5 dimensiones teóricas señaladas junto una vez obtenida la información se realizó el estadístico con el software STATA 14 obteniendo un Alfa de Cronbach de 0.75.

\section{Modelo de estimación de la calidad}

El modelo de SERVQUAL permite evaluar la percepción de la calidad, sin embargo, en cualquier modelo de estimación, es imprescindible contar con variables de control para determinar su relación con cada una de las dimensiones del modelo elegido. Al ser este modelo una estrategia recurrente en la literatura disponible para la medición de calidad de servicio se procedió a realizar una adecuación. Los estadísticos descriptivos se muestran en la siguiente tabla 2.

Tabla 2.

Descriptivos

\begin{tabular}{|l|l|l|l|l|}
\hline Variable & Mean & Std. Dev. & Min & Max \\
\hline Elementos tangibles & 3.7082 & 0.8661 & 1 & 5 \\
\hline Fiabilidad & 4.2376 & 0.8976 & 1 & 5 \\
\hline Capacidad de respuesta & 4.4324 & 0.8098 & 1 & 5 \\
\hline Seguridad & 4.5635 & 0.7621 & 1 & 5 \\
\hline Empatía & 3.9023 & 0.9366 & 1 & 5 \\
\hline
\end{tabular}

Fuente: Elaboración propia.

Acto seguido, se procedió a realizar un análisis correlacional de Pearson para establecer los niveles de significatividad de las dimensiones. La siguiente tabla 3 muestra estos datos.

Tabla 3.

Correlación Pearson

\begin{tabular}{|l|l|l|l|l|l|}
\hline Variables & E. TANG & FIAB & CAP.RESP. & SEG & EMPAT \\
\hline E. TANG & 1.0000 & & & & \\
\hline FIAB & $0.6355^{*}$ & 1.0000 & & & \\
\hline CAP.RESP. & $0.5049^{*}$ & $0.8318^{*}$ & 1.0000 & & \\
\hline SEG & $0.4469^{*}$ & $0.7391^{*}$ & $0.8186^{*}$ & 1.0000 & \\
\hline EMPAT & $0.5048^{*}$ & $0.6187^{*}$ & $0.6710^{*}$ & $0.6005^{*}$ & 1.0000 \\
\hline
\end{tabular}

Fuente: Elaboración propia con base en STATA 14.

Nota: Significatividad al: $0.01 \% *$

Como se puede observar, todas las dimensiones resultaron significativas al $0.05 \%$, por lo que los datos están en condiciones de ser analizados mediante la regresión lineal. Posteriormente se evalúo el índice global de consistencia de alfa Cronbach el cual fue de 0,75; por lo cual se pudo aplicar el instrumento al ser consistente y fiable para medir la calidad del servicio de acuerdo con Pérez-Cruz (2012). Finalmente, se aplicó el 
análisis de regresión lineal multinominal y se generaron las gráficas de los efectos.

\section{RESULTADOS}

En base a las técnicas aplicadas, se pudo conocer y analizar el impacto que generan las dimensiones comentadas con la calidad. La siguiente tabla muestra cuáles de las cinco dimensiones contempladas en el modelo SERVQUAL, se relacionan de manera lineal con la calidad en el servicio percibida por los usuarios, los estudiantes.

Tabla 2.

Regresión lineal

\begin{tabular}{|l|c|c|c|c|}
\hline \multicolumn{1}{|c|}{ Variables } & Coeficientes & Error estándar & & $P$-valor \\
\hline E. TANG & 0.112906 & 3.207 & 0.035210 & $0.00145^{\text {** }}$ \\
\hline FIAB & 0.002527 & 0.052247 & 0.048 & 0.96144 \\
\hline CAP.RESP. & 0.089327 & 0.067771 & -1.318 & 0.18820 \\
\hline SEG & 0.008293 & 0.056103 & 0.148 & 0.88256 \\
\hline EMPAT & 0.173094 & 0.054722 & 3.163 & $0.00167^{\text {** }}$ \\
\hline Constante & -0.356030 & 0.147760 & -2.410 & $0.01640^{*}$ \\
\hline Observ. 465 & $\mathrm{R}^{2}: 0.6700$ & $\mathrm{~F}: 11.34$ & & \\
\hline
\end{tabular}

Fuente: Elaboración propia con base en STATA 14.

Nivel de significancia: $0^{* * *} ; 0.001^{* *} ; 0.01^{*} ; 0.05^{\circ} ; 0.1^{\prime \prime}$.

El análisis anterior muestra que, de las cinco dimensiones, capacidad de respuesta, tangibles, empatía, fiabilidad y seguridad, sólo dos de ellas, específicamente la dimensión los elementos tangibles y la empatía, se relacionan significativamente con la dependiente, calidad en el servicio. Estos resultados permiten explicar el $61 \%$ de la calidad en el servicio percibida, lo que se corrobora con el nivel de significatividad de la constante (0.01) el valor de $\mathrm{P}$ (0.016). Las siguientes gráficas permiten visualizar las tendencias positivas de las variables significativas. 


\section{E..Tang. effect plot}

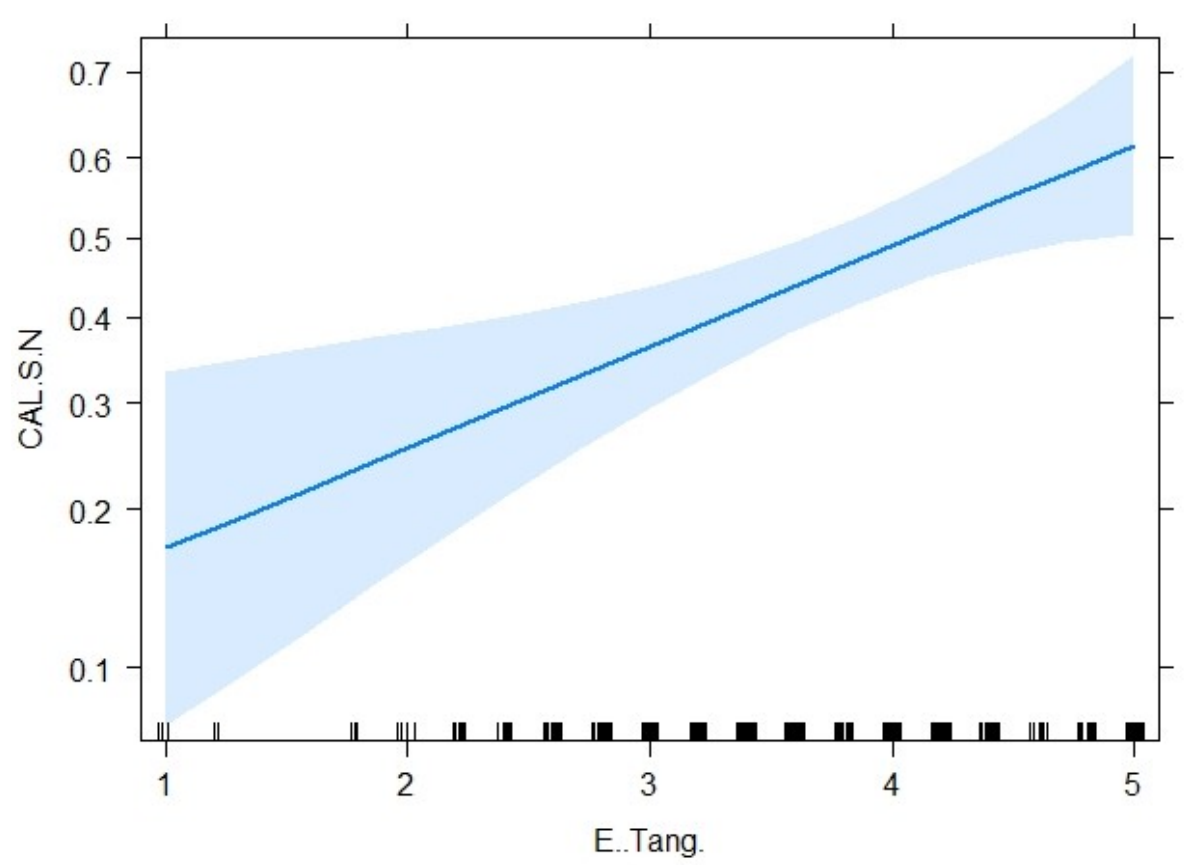

Gráfica 1.

Efectos de los elementos tangibles sobre la calidad en el servicio.

Fuente: Elaboración propia con base en STATA 14.

\section{Empat. effect plot}

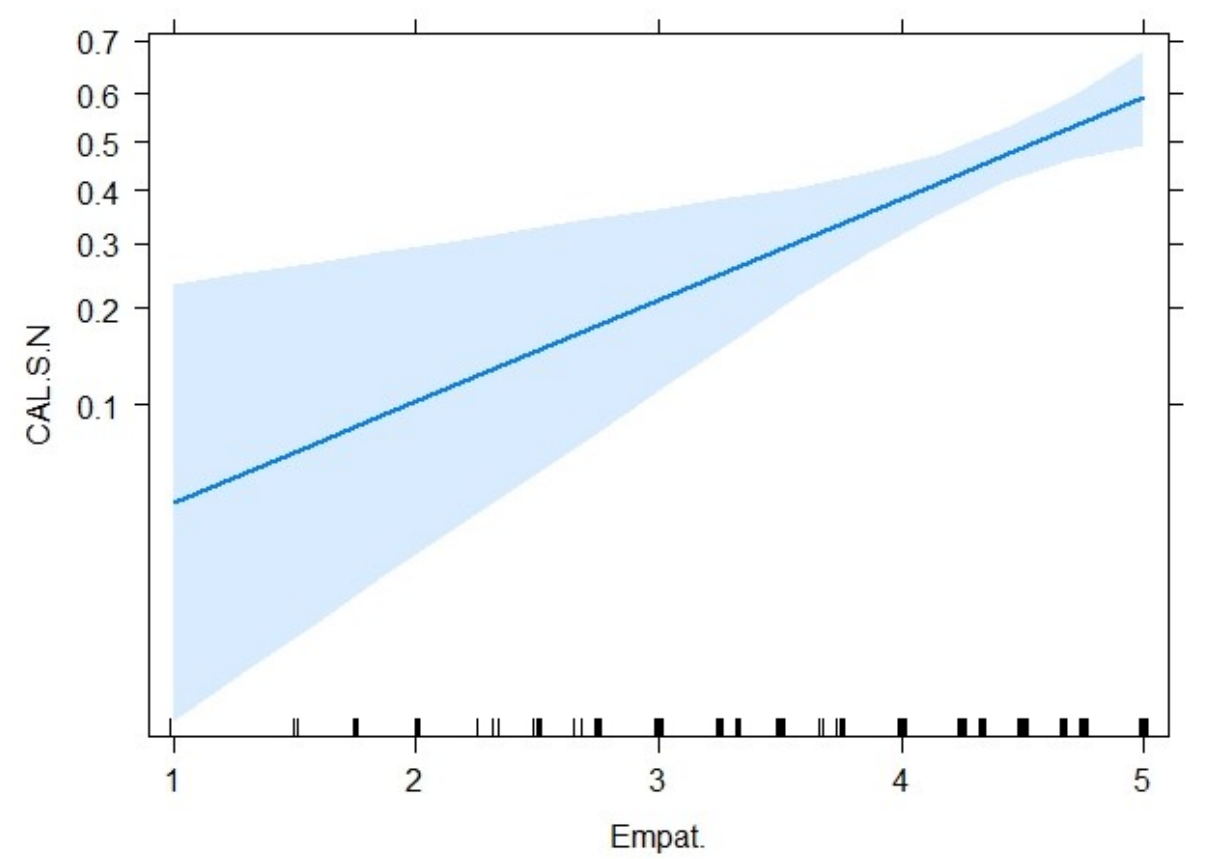

Gráfica 2.

Efectos de la empatía sobre la calidad en el servicio.

Fuente: Elaboración propia con base en STATA 14. 


\section{CONCLUSIONES}

La educación se ve reflejada como una necesidad latente entre los estudiantes, por lo que resalta la oportunidad de evaluar la calidad de los servicios que ofrece el proceso administrativo del SSC; actividad que se proporciona principalmente por la atención de personal administrativo de los planteles, ofreciendo un seguimiento personalizado en la atención del servicio prestado al estudiante. Con ello se reafirma la información obtenida en los resultados, donde las dimensiones de elementos tangibles y empatía destacan en su correlación a calidad.

El instrumento aplicado, permitió identificar los atributos más importantes para la evaluación de calidad de los servicios académicos y administrativos en la Dirección General de Servicio Social y Prácticas Profesionales (DGSSyPP), de la Universidad de Colima, con lo que se generó información para identificar las áreas de oportunidad en la prestación del servicio, y de esto modo, identificar los criterios de priorización de acciones en la planeación de sus procesos.

De este modo los elementos tangibles refuerzan uno de los puntos claves que respaldan la importancia de esta investigación: la capacidad instalada que tiene la institución para ofrecer el servicio y atender las necesidades de los clientes. En este punto, ante el requerimiento de cualquier servicio, es importante reconocer los beneficios que resultan en cuanto a: practicidad, agilidad y eficacia del servicio. Para los estudiantes el proceso de acreditación de SSC es un factor primordial para su desarrollo académico, marca una diferencia en su profesionalización, por la aplicación de los conocimientos adquiridos a lo largo de sus carreras, que lo lleven a fomentar una actitud proactiva y responsable en la toma de decisiones. También en la organización del tiempo y el poder relevar parte de la carga dependiente generada hacia las autoridades.

Por otra parte, la empatía logró posicionarse como una de las dimensiones significativas. Donde el estudiante resaltó: el valor de la atención, actitud e interés personalizado que se demuestra a través del servicio por parte del plantel o de las autoridades responsables; lo que para ellos significa una parte imprescindible de calidad durante el proceso. Esta dimensión representa una alta demanda por la comunidad estudiantil, lo que es lógico y normal, ya que se ven inmersos en un nuevo proceso que puede llegar a ser complejo. Esto, debido a tres escenarios diferentes que deben enfrentar: (1) La parte académica como materia dentro de su plan de estudios; (2) La parte administrativa para conseguir una acreditación; (3) La parte formativa que integra su capacitación profesional y un servicio en beneficio de la sociedad. Así pues, la actitud de las autoridades responsables es crucial para la satisfacción del usuario y para la atención de sus necesidades emergentes; lo que se ve reflejado en una orientación y asesoramiento puntual, personalizado en un ambiente empático para el estudiante.

En el mismo sentido, esta dimensión consideró como parte de los ítems relacionados a empatía, el evaluar el servicio y las instalaciones de la Dirección General de Servicio Social y Prácticas Profesionales 
(DGSSyPP) como dependencia responsable del proceso administrativo. El hecho de contemplar esta vertiente fue para averiguar tres situaciones: si el alumno realmente conoce la función de la DGSSyPP, si ha tenido contacto con la dependencia y si le brindaron un servicio de calidad. Estos datos permiten crear y reforzar un vínculo con los estudiantes, así como con sus necesidades. Lo anterior como parte de la calidad del servicio y como respaldo del mismo hacia las IES.

Como se mencionó, la evaluación constante en las IES contribuye a la detección de fortalezas y debilidades dentro del sistema universitario, y ayuda a la búsqueda continua de estrategias eficaces que permitan el avance y desarrollo institucional. La intención de elevar los estándares de calidad ya sea en servicios o productos, es ajustar y adaptarse a las nuevas necesidades del mercado estudiantil. El trabajo de los directivos implica conocer estas áreas de oportunidad para gestionar un cambio que detone soluciones innovadoras, ya sea en la estructura organizacional, administrativa o tecnológica, que logren transformarse en una ventaja competitiva.

\section{REFERENCIAS}

Cardona, M., y Bravo, J. (2012). Service quality perceptions in higher education institutions: the case of a colombian university. Estudios Gerenciales, 28(1), 23-29.

Cervantes, M. (2017). Higher Education Institutions in the Knowledge Triangle. Foresight and STI Governance, 11(2), 27-42. doi:https://doi.or $\mathrm{g} / 10.17323 / 2500-2597.2017 .2 .27 .42$

Duque, E., y Gómez, Y. (2015). Evolución conceptual de los modelos de medición de la percepción de calidad del servicio: Una mirada desde la educación superior. Suma de Negocios, 5(12), 180-191. doi:10.1016/ S2215-910X(14)70040-0

Flores, J., y Perez, O. (2006). Los costos de la no calidad en las pequeñas y medianas empresas. Contabilidad y Auditoria, 23(12), 1-27.

Geraldo, L. (2020). Valoración de la calidad del servicio educativo en instituciones educativas privadas. Industrial data, 23(1), 165-187. doi:10.15381/idata.v23i1.17178

Licona-Michel, A., y Perez-Cruz, O. (2018). El capital humano especializado en la estrategia de ciencia y tecnología en México. Praxis investigativa ReDIE: revista electrónica de la Red Durango de Investigadores Educativos, 10(19), 8-24.

Montaudon, C. (2010). Explorando la noción de la calidad. Acta Universitaria, 20(2), 50-56.

Parasuraman, A., Zeithalm, V., y Berry, L. (1985). A Conceptual Model of Service Quality and Its. Journal of Marketing, 49(1), 41-50.

Parasuraman, A., Zeithalm, V., y Berry, L. (1988). SERVQUAL: A multipleitem scale for measuring consumer perceptions of service quality. Journal of Retailing, 64(1), 12-40.

Perez-Cruz, O. (2012). Análisis de la seguridad laboral mediante la medición de los niveles de estrés laboral. Forum Empresarial, 7(2), 53-71. doi:https:// doi.org/10.33801/fe.v17i2.3749 
Pérez-Cruz, O. (2016). Procesos de desarrollo económico y sociocultural en el México del siglo XX. Colima: Universidad de Colima.

Perez-Cruz, O. (2018). Capitales sociales y trayectorias laborales. Administración y Organizaciones, 21(40), 229-244. doi: http://orcid.org/ 0000-0003-3367-8259

Pérez-Cruz, O. (2019). Innovación y transferencia de tecnología en México.Un análisis empírico de datos panel. RIDE. Revista Iberoamericana para la Investigación y el Desarrollo Educativo, 10(19), 200-2018. doi:https://doi .org/10.23913/ride.v10i19.503

Perez-Cruz, O., y Pinto-Perez, R. (2020). Determinants of Labor Insertion inUniversity Graduates in México. RIDE Revista Iberoamericana para la investigación y el Desarrollo Educativo, 11(21), 1-16. doi:https://doi.org/ 10.23913/ride.v11i21.732

Reyes, T., Nande, E., y Hernández, L. (2020). Factores determinantes de la productividad en las universidades públicas mexicanas. Lider, 22(36), 89-103. doi:https://doi.org/10.32735/S0719-52652020364

Sotelo, G., y Figueroa, E. (2017). El clima organizacional y su correlación con la calidad en el servicio en una institución de educación de nivel medio superior. RIDE. Revista Iberoamericana para la Investigación y el Desarrollo Educativo, 8(15), 582-609. doi:https://doi.org/10.23913/ride .v8i15.312

Wang, Y. (2015). Contribution and Trend to Quality Research-a literature review of SERVQUAL model from 1998 to 2013. Informatica economica, 19(1), 34-45. doi:0.12948/issn14531305/19.1.2015.03 